\title{
Dopplervelocimetria fetoplacentária em gestantes hipertensas e resultados perinatais segundo a idade gestacional*
}

\author{
Fetal and placental Doppler velocimetry in hypertensive pregnant women and perinatal \\ outcomes according to gestational age
}

Pedro Pires ${ }^{1}$, Aníbal Eusébio Faúndes Latham ${ }^{2}$, Suellene Keylla de Magalhães Mabessone ${ }^{3}$, Ana de Fátima de Azevedo Ferreira ${ }^{3}$, Fabiana Gomes de Souza Rodrigues ${ }^{3}$, Janaina Souza Leon $^{3}$, Juliana Limeira de Moura Ramos ${ }^{3}$

Resumo OBJETIVO: Avaliar índices de pulsatilidade das artérias umbilical (IPAU) e cerebral média (IPACM) e relação do índice de pulsatilidade umbilico-cerebral (IPAU/IPACM) em fetos de gestantes hipertensas e presença de resultados perinatais adversos. MATERIAIS E MÉTODOS: Analisamos IPAU, IPACM e IPAU/IPACM de 289 fetos de gestantes hipertensas quanto à previsão dos resultados perinatais adversos. Os resultados foram comparados sem e com ajuste pela idade gestacional. RESULTADOS: 0 índice de Apgar $<7$ no $5^{\circ}$ minuto foi associado com resultados alterados após o ajuste por idade gestacional. 0 risco para recém-nascidos pequenos para a idade gestacional aumentou em três vezes após o ajuste, com significância estatística em todos os parâmetros do Doppler. Na síndrome da hipóxia neonatal o aumento do risco ajustado pela idade gestacional foi estatisticamente significante no IPAU e IPAU/IPACM. Não houve aumento no risco de síndrome do desconforto respiratório na análise ajustada. A mortalidade perinatal e o IPAU alterado apresentaram um risco três vezes maior e foram estatisticamente significantes após o ajuste. CONCLUSÃO. Em gestantes hipertensas, o IPAU apresentou melhor correlação com os resultados perinatais do que o IPACM ou relação IPAU/IPACM. O risco de resultados adversos deve considerar a idade gestacional.

Unitermos: Dopplervelocimetria; Hipertensão; Resultados perinatais.

Abstract OBJECTIVE: To evaluate the pulsatility index of umbilical artery (UAPI) and middle cerebral artery (MCAPI), as well as the umbilical-cerebral pulsatility (UAPI/MCAPI) ratio in fetuses of hypertensive pregnant women and associated adverse perinatal outcomes. MATERIALS AND METHODS: The authors have analyzed UAPI, MCAPI and UAPI/MCAPI ratio in 289 fetuses of hypertensive women, correlating the results with the presence of adverse perinatal outcomes. Results were compared with and without adjustment for gestational age. RESULTS: Apgar score $<7$ at the 5th minute was associated with altered outcomes after adjustment for gestational age. The risk for small-for-gestational-age infant increased three times after such adjustment, with statistical significance for all the Doppler parameters. The increase in risk for neonatal hypoxia after adjustment for gestational age was statistically significant for UAPI and UAPI/MCAPI ratio. No increase was observed in the risk for respiratory distress syndrome in the adjusted analysis. A three-time higher risk for perinatal mortality and altered UAPI with statistical significance was observed after adjustment. CONCLUSION: In fetuses of hypertensive pregnant women, UAPI demonstrated better correlations with perinatal outcomes than MCAPI and UAPI/MCAPI ratio. The risk for adverse gestational outcome should be evaluated taking the gestational age into consideration.

Keywords: Doppler velocimetry; Hypertension; Perinatal outcome.

Pires P, Latham AEF, Mabessone SKM, Ferreira AFA, Rodrigues FGS, Leon JS, Ramos JLM. Dopplervelocimetria fetoplacentária em gestantes hipertensas e resultados perinatais segundo a idade gestacional. Radiol Bras. 2010;43(3):155-160.

* Trabalho realizado na Faculdade de Ciências Médicas da Universidade de Pernambuco (UPE), Recife, PE, Brasil.

1. Doutor, Coordenador do Serviço de Medicina Fetal do Departamento Materno Infantil da Faculdade de Ciências Médicas da Universidade de Pernambuco (UPE), Recife, PE, Brasil.

2. Doutor, Professor Colaborador Voluntário de Obstetrícia da Universidade Estadual de Campinas (Unicamp), Campinas, SP, Brasil.

3. Especialistas em Ultrassonografia e Tocoginecologia, Médicas Obstetras do Departamento Materno Infantil da Faculdade de Ciências Médicas da Universidade de Pernambuco (UPE), Recife, PE, Brasil.

Endereço para correspondência: Dr. Pedro Pires Ferreira Neto.

\section{INTRODUÇÃO}

Uma das principais preocupações da obstetrícia atual é a de assegurar-se das boas condições de vitalidade do feto de risco. Esta preocupação é ainda maior quando

Avenida Doutor Malaquias, 195, ap. 1401, Graças. Recife, PE, Brasil, 52050-060.E-mail:nppires@uol.com.br

Recebido para publicação em 16/6/2009. Aceito, após revisão, em 11/3/2010. existem doenças maternas que sabidamente podem causar insuficiência placentária e crescimento intrauterino restrito (CIUR), como é o caso, principalmente, da hipertensão arterial ${ }^{(\mathbf{1})}$. Esta é a entidade clínica que mais se associa ao CIUR e à insuficiência placentária $^{(2-7)}$.

A técnica Doppler possibilita o estudo da circulação uterina e fetoplacentária, de forma não invasiva, permitindo diagnosticar 
precocemente estados de hipóxia e predizer resultados perinatais adversos ${ }^{(\mathbf{6 , 8}-10)}$.

O exame Doppler permite analisar os índices de resistência nos principais vasos, sendo os mais utilizados a artéria umbilical e a artéria cerebral média ${ }^{(\mathbf{1 1}-14)}$. Na fase compensada da hipóxia a resistência placentária aumenta e as artérias umbilicais elevam seus índices de resistência. Na sequência se observa redução progressiva da resistência vascular cerebral, evoluindo para a "centralização". Este fenômeno antecede em 10 a 12 dias o grave comprometimento fetal, com acidose fetal e maior morbimortalidade perinatal ${ }^{(\mathbf{6})}$.

Alterações no padrão Doppler na artéria umbilical, especialmente a diástole ausente ou reversa, são indicativos de disfunção placentária ${ }^{(\mathbf{1 5})}$, sendo estes fetos de alto risco para sofrimento fetal e particularmente vulneráveis para as complicações da prematuridade $^{(\mathbf{1 6})}$, o que determina um equilíbrio entre o risco fetal versus o risco neonatal na identificação do momento apropriado da intervenção ${ }^{(\mathbf{1 7 - 1 9 )}}$

O manejo obstétrico, para alguns autores, ainda é predominantemente baseado na análise Doppler da artéria umbilical ${ }^{(\mathbf{4}, \mathbf{1 8}}$, 20-22), embora alguns estudos direcionem para a inclusão de mais de um vaso na avaliação da vitalidade dos fetos de $\operatorname{risco}^{(7,23)}$

Vale ressaltar que as alterações graves dopplervelocimétricas, indicativas de vigilância fetal intensiva ou de interrupção, ocorrem em fetos prematuros, particularmente na faixa da prematuridade extrema ${ }^{(24)}$

Com o propósito de esclarecer a capacidade de distintos indicadores do Doppler de predizer o risco de complicações perinatais, estudamos, simultaneamente, o risco de maus resultados perinatais quando existem alterações dopplervelocimétricas da artéria umbilical, cerebral média e índice umbilico-cerebral. Ao mesmo tempo, verificamos se os resultados encontrados permanecem ou mudam substancialmente após controlar pela idade gestacional no parto.

\section{MATERIAIS E MÉTODOS}

A série inicial foi composta de 497 gestantes hipertensas, no período de janeiro de 2002 a agosto de 2006. Para o estudo da circulação fetoplacentária empregou-se equipamento de ultrassonografia da marca
Shimadzu, modelo SDU-2200 (Shimadzu; Kyoto, Japão), com dispositivo Doppler e mapeamento colorido do fluxo sanguíneo e transdutor convexo de $3,5 \mathrm{MHz}$. O filtro de janela foi fixado entre 50 e $100 \mathrm{~Hz}$. Com a gestante acomodada em posição semiFowler e na ausência de movimentação corporal e respiratória fetal, utilizando imagens em tempo real, acionou-se o mapeamento em cores do fluxo sanguíneo, obtendo-se mapeamento colorido dos vasos a serem estudados, que foram avaliados utilizando-se o dispositivo Doppler, com ajustes do volume de amostra para cada vaso, na ausência de movimentos fetais.

O Doppler da artéria umbilical foi realizado em alça livre o mais próximo da inserção placentária e o ângulo de insonação sempre menor que $60^{\circ}$. A análise espectral foi considerada adequada quando exibiu pelo menos três ondas de velocidade semelhantes no mesmo espectro (Figura 1). Quando, na artéria umbilical, a diástole esteve ausente ou reversa ou o índice de pulsatilidade esteve acima do percentil 95 , foi considerado anormal ${ }^{(25)}$.

A artéria cerebral média foi visualizada a partir do polígono de Willis e insonada imediatamente após sua origem na artéria carótida interna. O ângulo entre o feixe sonoro e o fluxo foi captado o mais próximo possível de $0^{\circ}$, sendo mensurado o índice de pulsatilidade (Figura 2). O índice de pulsatilidade da artéria cerebral média (IPACM) abaixo do percentil 5 e a relação do índice de pulsatilidade da artéria umbilical/artéria cerebral média (IPAU/IPACM) foram considerados anormais se a medida foi acima do percentil 95 para a idade gestacional $^{(25)}$.

Foram excluídos os casos com intervalo entre o último exame dopplervelocimétrico e o parto maior que 7 dias, não obtenção dos resultados perinatais no nascimento, defeito congênito ou anormalidade cromossômica fetal ou neonatal, doença materna responsável por infecção congênita e idade gestacional no momento do parto inferior a 24 semanas. Aplicando estes critérios, foram excluídas 208 gestantes, ficando a amostra final com um total de 289 gestantes que preencheram os critérios de inclusão.

Os dados foram organizados em programa Epi-Info, versão 1.0. Os casos foram distribuídos em três faixas de idade gestacional ( $<33$ semanas, 33 a 36 semanas e $\geq 37$ semanas) e classificados segundo a presença ou ausência de cada resultado perinatal adverso: Apgar no $5^{\circ}$ minuto $<7$, recém-nascido pequeno para a idade gestacional (PIG), ocorrência de síndrome hipó-

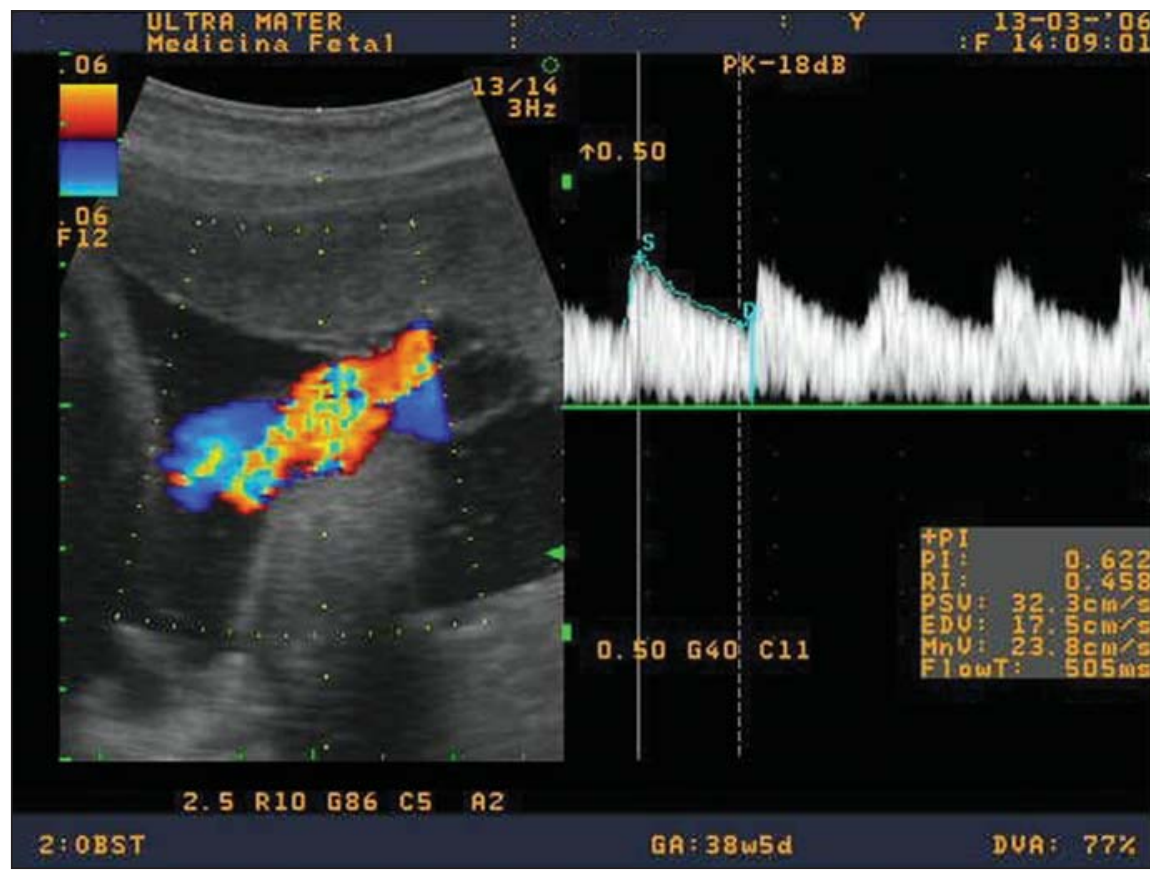

Figura 1. Dopplenvelocimetria da artéria umbilical próximo da inserção placentária. 


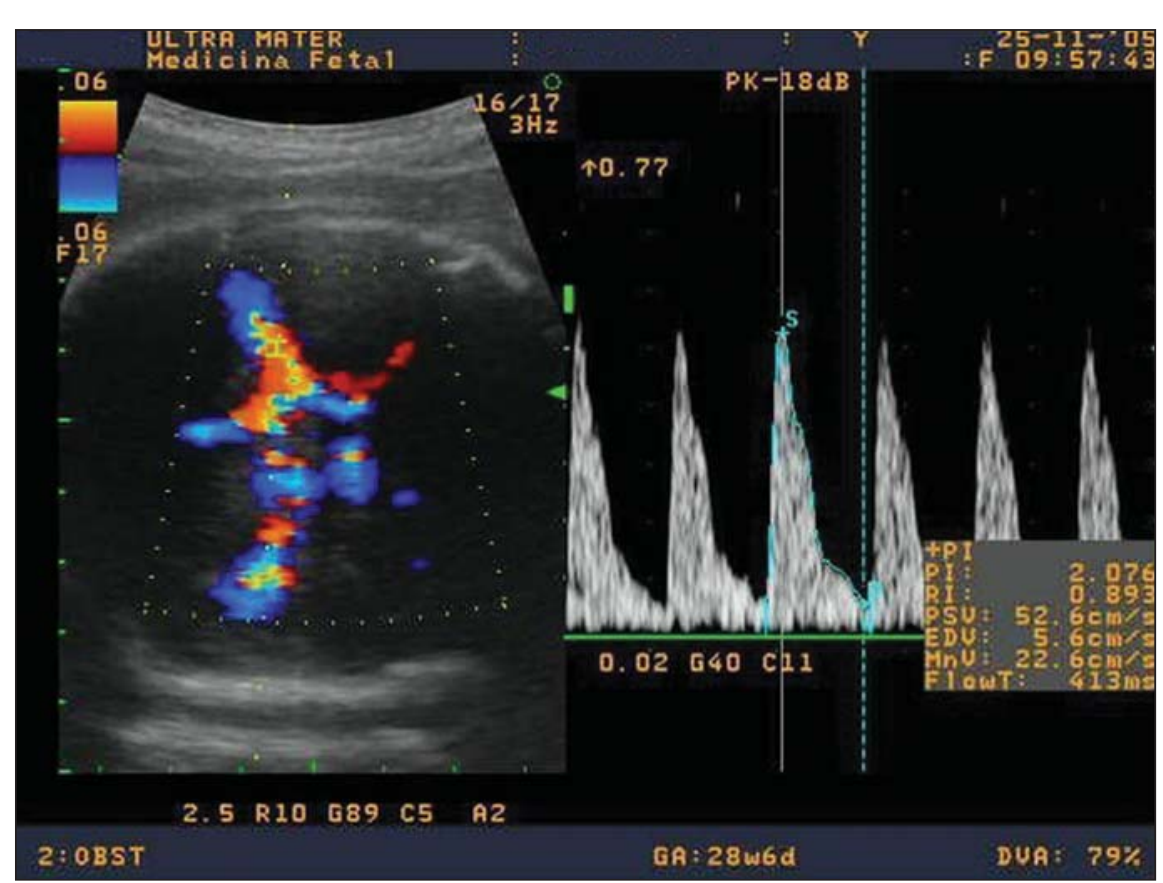

Figura 2. Dopplervelocimetria da artéria cerebral média visualizada a partir do polígono de Willis e insonada imediatamente após sua origem na artéria carótida interna. 0 ângulo entre o feixe sonoro e o fluxo foi captado o mais próximo possível de $0^{\circ}$.

xico-isquêmica (SHI) neonatal, ocorrência de síndrome do desconforto respiratório (SDR) e óbito perinatal.

Realizou-se análise prospectiva de avaliação do risco de maus resultados perinatais calculado pelo risco relativo estimado (odds ratio) dos desfechos estudados, segundo os resultados das dopplervelocimetrias realizadas no máximo até 7 dias antes do parto. Posteriormente, se repetiu o mesmo cálculo, ajustado segundo a idade gestacional.

O presente estudo atende à Resolução 196/1996 do Conselho Nacional de Saúde e às recomendações da Declaração de Helsinki VI para pesquisa em seres humanos, tendo sido submetido e aprovado pela Comissão de Ética em Pesquisa do Centro Integrado de Saúde Amaury de Medeiros da Universidade de Pernambuco.

\section{RESULTADOS}

\section{Características da amostra}

A idade das mulheres variou entre $14 \mathrm{e}$ 43 anos (idade média de 26 anos). Quanto à paridade, $57,1 \%$ das gestantes eram primigestas. Quase dois terços dos casos corresponderam a pré-eclâmpsia. A via de parto foi vaginal em apenas $25,6 \%$ dos

Tabela 1 Características das 289 gestantes. de morte fetal e $6,5 \%$ de mortes neonatais (Tabela 2).

\section{Associação entre o exame dopplervelocimétrico e os resultados perinatais}

Não se constatou associação do IPAU, IPACM e da relação IPAU/IPACM com o índice de Apgar $<7$ no $5^{\circ}$ minuto, antes de ajustar por idade gestacional. Após ajustar, o risco de Apgar $<7$ no $5^{\circ}$ minuto foi mais de duas vezes e quase duas vezes maior em fetos em que o IPAU e o IPACM estavam alterados, respectivamente. Entretanto, apenas a associação com alteração do IPAU teve significância estatística (Tabela 3).

Verificou-se que a alteração do IPAU, IPACM e IPAU/IPACM aumentou o risco de ocorrência de recém-nascidos PIG entre cinco e sete vezes na análise sem ajuste e em torno de três vezes na análise ajustada segundo idade gestacional (Tabela 4). O aumento do risco foi estatisticamente significativo para todos os parâmetros da dopplervelocimetria estudados.

Alterações do IPAU, IPACM e da relação IPAU/IPACM aumentaram entre duas e cinco vezes o risco de SHI antes do ajuste, e entre uma e meia vez e mais de três e meia vezes após ajuste por idade gestacional. $\mathrm{O}$ aumento do risco ajustado foi significativo apenas para IPAU e relação IPAU/IPACM (Tabela 5).

Observou-se aumento de duas a seis vezes do risco de SDR nos casos com IPAU, IPACM e relação IPAU/IPACM alterados,

\begin{tabular}{lcc}
\hline & $n$ & Percentual \\
\hline Paridade & & $57,1 \%$ \\
Para I & 165 & $33,2 \%$ \\
Para II e III & 96 & $9,7 \%$ \\
Acima de III & 28 & $23,9 \%$ \\
Tipo de hipertensão & 69 & $63,0 \%$ \\
Hipertensão arterial sistêmica & 182 & $13,1 \%$ \\
Pré-eclâmpsia & 38 & $25,6 \%$ \\
Pré-eclâmpsia superposta & & $59,5 \%$ \\
Via de parto & 74 & $14,9 \%$ \\
Vaginal & 172 & \\
Cesárea eletiva & 43 & $51,2 \%$ \\
Cesárea intraparto & & $24,6 \%$ \\
Intervalo: último exame Doppler e o parto & 148 & $24,2 \%$ \\
Um dia & 71 & 70 \\
Entre 2 e 3 dias & 73 & \\
Entre 4 e 7 dias & & \\
\hline
\end{tabular}


Tabela 2 Resultados perinatais.

\begin{tabular}{lcc}
\hline & $n$ & Percentual \\
\hline Idade gestacional & & \\
$<33$ semanas & 80 & $27,7 \%$ \\
33 a 36 semanas & 95 & $32,9 \%$ \\
$\geq 37$ semanas & 114 & $39,4 \%$ \\
Faixa de peso do recém-nascido em gramas (g) & & \\
$<1.500 \mathrm{~g}$ & 53 & $18,4 \%$ \\
1.500 a $2.499 \mathrm{~g}$ & 85 & $29,4 \%$ \\
$\geq 2.500 \mathrm{~g}$ & 151 & $52,2 \%$ \\
Apgar no 5० minuto $<7$ & 61 & $21,5 \%$ \\
Recém-nascido pequeno para a idade gestacional & 91 & $31,5 \%$ \\
Síndrome hipóxico-isquêmica & 56 & $19,8 \%$ \\
Síndrome do desconforto respiratório & 46 & $16,3 \%$ \\
Óbito perinatal & & \\
$\quad$ Morte fetal & 5 & $2,0 \%$ \\
$\quad$ Mortes neonatais & 16 & $6,5 \%$ \\
\hline
\end{tabular}

Tabela 3 Risco relativo estimado (odds ratio) de índice de Apgar $<7$ aos 5 minutos, sem ajuste e após ajuste por idade gestacional, segundo o indicador alterado da dopplenvelocimetria.

\begin{tabular}{lcccc}
\hline Indicador alterado & Odds ratio bruto & IC 95\% & Odds ratio ajustado & IC 95\% \\
\hline IPAU & 1,31 & $(0,55-2,89)$ & 2,65 & $(1,03-6,85)$ \\
IPACM & 1,36 & $(0,71-2,57)$ & 1,85 & $(0,96-3,58)$ \\
IPAU/IPACM & 0,89 & $(0,44-1,37)$ & 1,45 & $(0,66-5,99)$
\end{tabular}

IPAU, índice de pulsatilidade da artéria umbilical; IPACM, índice de pulsatilidade da artéria cerebral média; IPAU/ IPACM, relação do índice de pulsatilidade da artéria umbilical/artéria cerebral média.

Tabela 4 Risco relativo estimado (odds ratio) de pequeno para idade gestacional, sem ajuste e após ajuste por idade gestacional, segundo o indicador alterado da dopplervelocimetria.

\begin{tabular}{lcccc}
\hline Indicador alterado & Odds ratio bruto & IC 95\% & Odds ratio ajustado & IC 95\% \\
\hline IPAU & 7,48 & $(3,57-16,09)$ & 2,98 & $(1,36-6,56)$ \\
IPACM & 5,43 & $(3,05-9,70)$ & 3,24 & $(1,78-5,89)$ \\
IPAU/IPACM & 7,37 & $(4,06-13,41)$ & 3,15 & $(1,66-5,99)$ \\
\hline
\end{tabular}

IPAU, índice de pulsatilidade da artéria umbilical; IPACM, índice de pulsatilidade da artéria cerebral média; IPAU/ IPACM, relação do índice de pulsatilidade da artéria umbilical/artéria cerebral média.

Tabela 5 Risco relativo estimado (odds ratio) de síndrome hipóxico-isquêmica do recém-nascido, sem ajuste e após ajuste por idade gestacional, segundo o indicador alterado da dopplervelocimetria.

\begin{tabular}{lcccc}
\hline Indicador alterado & Odds ratio bruto & IC 95\% & Odds ratio ajustado & IC 95\% \\
\hline IPAU & 4,93 & $(2,30-10,43)$ & 3,66 & $(1,77-7,57)$ \\
IPACM & 2,36 & $(1,23-4,49)$ & 1,54 & $(0,80-2,99)$ \\
IPAU/IPACM & 4,49 & $(2,32-8,68)$ & 2,79 & $(1,34-5,82)$
\end{tabular}

IPAU, índice de pulsatilidade da artéria umbilical; IPACM, índice de pulsatilidade da artéria cerebral média; IPAU/ IPACM, relação do índice de pulsatilidade da artéria umbilical/artéria cerebral média.

Tabela 6 Risco relativo estimado (odds ratio) de síndrome do desconforto respiratório, sem ajuste e após ajuste por idade gestacional, segundo o indicador alterado da dopplenvelocimetria.

\begin{tabular}{lcccc}
\hline Indicador alterado & Odds ratio bruto & IC 95\% & Odds ratio ajustado & IC 95\% \\
\hline IPAU & 6,06 & $(2,69-13,39)$ & 1,56 & $(0,68-3,60)$ \\
IPACM & 2,35 & $(1,15-4,74)$ & 1,04 & $(0,48-2,25)$ \\
IPAU/IPACM & 3,93 & $(1,92-8,03)$ & 0,90 & $(0,38-2,09)$
\end{tabular}

IPAU, índice de pulsatilidade da artéria umbilical; IPACM, índice de pulsatilidade da artéria cerebral média; IPAU/ IPACM, relação do índice de pulsatilidade da artéria umbilical/artéria cerebral média. na análise sem ajuste. No entanto, na análise ajustada por idade gestacional não houve aumento do risco desta complicação neonatal nos casos com essas alterações no Doppler (Tabela 6).

Apesar de o risco bruto de mortalidade perinatal ter sido de três a dez vezes maior nos casos com alteração do IPAU, IPACM e da relação IPAU/IPACM, na análise ajustada segundo a idade gestacional apenas o IPAU alterado apresentou risco de quase três vezes maior de morte perinatal estatisticamente significante (Tabela 7).

Na Tabela 8 são expostos os resultados resumidos para melhor visualização e comparação dos distintos indicadores de alteração do Doppler. Nota-se que a artéria umbilical é o melhor indicador de risco de mau resultado perinatal, particularmente quando a avaliação do risco se faz ajustando por idade gestacional do recém-nascido.

\section{DISCUSSÃO}

Os resultados em nosso estudo sugerem que a avaliação do fluxo sanguíneo da artéria umbilical foi o melhor indicador de risco de maus resultados perinatais, em comparação com a avaliação da artéria cerebral média e com o índice umbilico-cerebral. Os três indicadores parecem ter, antes de ajustar pela idade gestacional, uma capacidade semelhante de predizer maus resultados perinatais. É importante frisar que o aumento de risco foi sempre maior quando se considera o Doppler da artéria umbilical comparado com a artéria cerebral média. Vale destacar que a diferença entre estes dois indicadores é mais evidente quando observamos os riscos de maus resultados perinatais após ajuste pela idade gestacional. Vários autores não consideraram, em seus estudos, o fator idade gesta-

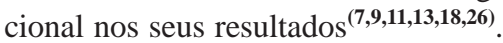

As alterações do IPAU, em nossa análise, permitiram predizer maior risco (próximo a três vezes) em quatro dos cinco indicadores de resultados perinatais adversos estudados. Para a SDR, o risco foi $50 \%$ maior, mas não chegou a ser significativo. Em contraste, alteração do IPACM permitiu predizer maior risco apenas de PIG, e a relação IPAU/IPACM, apenas de PIG e SHI.

Para alguns autores, o estudo de mais de um vaso contribui para aprimorar o diag- 
Tabela 7 Risco relativo estimado (odds ratio) de morte perinatal, sem ajuste e após ajuste por idade gestacional, segundo o indicador alterado da dopplervelocimetria.

\begin{tabular}{lcccc}
\hline Indicador alterado & Odds ratio bruto & IC 95\% & Odds ratio ajustado & IC 95\% \\
\hline IPAU & 9,84 & $(3,80-25,67)$ & 2,87 & $(1,10-7,45)$ \\
IPACM & 2,81 & $(1,15-6,98)$ & 1,18 & $(0,48-2,94)$ \\
IPAU/IPACM & 6,08 & $(2,37-16,08)$ & 1,57 & $(0,56-4,35)$ \\
\hline
\end{tabular}

IPAU, índice de pulsatilidade da artéria umbilical; IPACM, índice de pulsatilidade da artéria cerebral média; IPAU/ IPACM, relação do índice de pulsatilidade da artéria umbilical/artéria cerebral média.

Tabela 8 Risco relativo estimado de maus resultados perinatais segundo o indicador de alteração na dopplervelocimetria.

\begin{tabular}{lccc}
\hline & \multicolumn{3}{c}{ Indicador de alteração do Doppler } \\
\cline { 2 - 4 } & $\begin{array}{c}\text { Artéria } \\
\text { umbilical }\end{array}$ & $\begin{array}{c}\text { Artéria cerebral } \\
\text { média }\end{array}$ & Índice U/C \\
\hline Sem ajustar & & & \\
Apgar no $5^{\circ}$ minuto $<7$ & $(1,31)^{*}$ & $(1,36)$ & $(0,89)$ \\
Recém-nascido pequeno para a idade gestacional & 7,48 & 5,43 & 7,37 \\
Síndrome hipóxico-isquêmica & 4,93 & 2,36 & 4,49 \\
Síndrome do desconforto respiratório & 6,06 & 2,35 & 3,93 \\
Morte perinatal & 9,84 & 2,81 & 6,08 \\
Ajustado por idade gestacional & & & \\
Apgar no 50 minuto $<7$ & 2,65 & $(1,85)$ & $(1,45)$ \\
Recém-nascido pequeno para a idade gestacional & 2,98 & 3,24 & 3,15 \\
Síndrome hipóxico-isquêmica & 3,66 & $(1,54)$ & 2,79 \\
Síndrome do desconforto respiratório & $(1,56)$ & $(1,04)$ & $(0,90)$ \\
Morte perinatal & 2,87 & $(1,18)$ & $(1,57)$ \\
\hline
\end{tabular}

* Valores entre parênteses não foram estatisticamente significantes.

nóstico da situação fetal, que avalia a capacidade da dopplervelocimetria em prever os resultados perinatais, incluindo apenas fetos com diagnóstico firmado de CIUR $^{(3,}$ 6,7,12,27,28). Em nosso estudo incluímos gestantes hipertensas, que pode levar a insuficiência placentária e potencialmente representa um risco de dano fetal, mas sem que este necessariamente esteja presente.

Outro dado importante está relacionado em avaliar os resultados perinatais em casos com diástole zero ou fluxo reverso, que são alterações graves no padrão dopplervelocimétrico ${ }^{(\mathbf{9}, 10,13,26)}$. Em nosso estudo consideramos não apenas a diástole ausente ou reversa como resultados alterados na artéria umbilical, mas, também, os casos em que o índice de pulsatilidade esteve acima do percentil 95 .

Outro fator considerado importante na análise do nosso estudo foi que aproximadamente $75 \%$ de nossos casos tiveram um intervalo entre o exame e o parto de até três dias e 50\%, de um dia. Estudo realizado por Baschat et al. ${ }^{(7)}$ não informou o intervalo médio entre o último exame e o parto, en- quanto para outros autores o intervalo médio foi além de sete dias ${ }^{(\mathbf{9 , 2 9 )}}$. Variações no intervalo exame-parto podem explicar diferenças nos resultados, visto que as alterações graves do padrão Doppler das artérias umbilical e cerebral antecedem em 10 a 14 dias o agravamento do status fetal ${ }^{(6)}$.

A prematuridade permanece como o maior determinante de mortalidade neonatal e das suas complicações ${ }^{(9,27,28,30)}$. Há autores que analisaram apenas os fetos prematuros, principalmente com idade gestacional abaixo de 32 semanas, diferentemente dos casos do nosso estudo, em que incluímos distintas idades gestacionais, o que permitiu analisarmos separadamente os resultados em prematuros extremos, prematuros e fetos a termo, e avaliarmos o impacto da prematuridade nos resultados perinatais ${ }^{(3,7,11,13)}$.

A mudança nos resultados obtidos após corrigirmos pela idade gestacional foi significativa. Sabemos que, ao se fazer o diagnóstico de insuficiência placentária e alterações hemodinâmicas fetais, a tendência é a de interromper a gestação para evitar a morte fetal intrauterina. É evidente, portanto, a associação entre alterações no Doppler e menor idade gestacional. Como sabemos que quanto menor a idade gestacional, piores os resultados perinatais, entende-se que a idade gestacional seja uma variável confundidora no estudo da associação entre alterações no Doppler e resultados perinatais.

O exemplo mais típico é o da SDR. Antes de ajustar por idade gestacional, as alterações do Doppler da artéria umbilical apareciam associadas a um risco seis vezes maior de SDR, mas após o ajuste essa associação passou a ser não significativa. De todos os indicadores de maus resultados perinatais estudados, a SDR é justamente a mais estreitamente relacionada com a idade gestacional, mais ainda do que a situação de hipoxemia crônica intrauterina. Entende-se, portanto, que o aumento do risco de SDR em casos com alteração no Doppler se deva muito mais à interrupção prematura da gestação induzida pelo resultado do Doppler do que a situação de hipóxia do feto.

Seria muito atrativo se a avaliação do status fetal fosse mais importante do que os efeitos da idade gestacional nos resultados perinatais; desta forma, o momento do parto poderia ser baseado nos testes de avaliação fetal.

Acreditamos, portanto, ser de extrema importância a observação de que não devemos ignorar a idade gestacional diante dos dados analisados. Esta foi uma das contribuições mais importantes deste estudo. Confiamos que a publicação destes resultados contribuirá para que estudos futuros possam confirmar a importância do fator idade gestacional em suas análises à realização da dopplervelocimetria na avaliação da vitalidade fetal.

\section{REFERÊNCIAS}

1. Sebire NJ. Umbilical artery Doppler revisited: pathophysiology of changes in intrauterine growth restriction revealed. Ultrasound Obstet Gynecol. 2003;21:419-22.

2. Campbell S, Pearce JM, Hackett G, et al. Qualitative assessment of uteroplacental blood flow: early screening for high-risk pregnancies. Obstet Gynecol. 1986;68:649-53.

3. Ozcan T, Sbracia M, d'Ancona RL, et al. Arterial and venous Doppler velocimetry in the severely growth-restricted fetus and associations with adverse perinatal outcome. Ultrasound Obstet Gynecol. 1998;12:39-44.

4. Nomura RMY, Francisco RPV, Sakamoto K, et al. Centralização da circulação fetal em gestações de 
alto risco: avaliação da vitalidade fetal e resultados perinatais. Rev Bras Ginecol Obstet. 2001; 23:137-43.

5. Sebire NJ, Goldin RD, Regan L. Histomorphological evidence for chronic vasoconstriction of placental stem vessels in pregnancies with intrauterine growth restriction and abnormal umbilical artery Doppler velocimetry indices. J Pathol. 2001;195:19A.

6. Baschat AA, Gembruch U, Weiner CP, et al. Qualitative venous Doppler waveform analysis improves prediction of critical perinatal outcomes in premature growth-restricted fetuses. Ultrasound Obstet Gynecol. 2003;22:240-5.

7. Baschat AA, Galan HL, Bhide A, et al. Doppler and biophysical assessment in growth restricted fetuses: distribution of test results. Ultrasound Obstet Gynecol. 2006;27:41-7.

8. Soothill PW, Ajayi RA, Campbell S, et al. Relationship between fetal acidemia at cordocentesis and subsequent neurodevelopment. Ultrasound Obstet Gynecol. 1992;2:80-3.

9. Bilardo CM, Wolf H, Stigter RH, et al. Relationship between monitoring parameters and perinatal outcome in severe, early intrauterine growth restriction. Ultrasound Obstet Gynecol. 2004;23: $119-25$.

10. Hartung J, Kalache KD, Heyna C, et al. Outcome of 60 neonates who had ARED flow prenatally compared with a matched control group of appropriate-for-gestational age preterm neonates. U1trasound Obstet Gynecol. 2005;25:566-72.

11. Hecher K, Bilardo CM, Stigter RH, et al. Monitoring of fetuses with intrauterine growth restriction: a longitudinal study. Ultrasound Obstet Gynecol. 2001;18:564-70.

12. Harman CR, Baschat AA, Gembruch U. Venous Doppler in IUGR. Which vessel? Which parameter? Am J Obstet Gynecol. 2001;185:53.

13. Ferrazzi E, Bozzo M, Rigano S, et al. Temporal sequence of abnormal Doppler changes in the peripheral and central circulatory systems of the severely growth-restricted fetus. Ultrasound Obstet Gynecol. 2002;19:140-6.

14. Schreuder AM, McDonnell M, Gaffney G, et al. Outcome at school age following antenatal detection of absent or reversed end diastolic flow velocity in the umbilical artery. Arch Dis Child Fetal Neonatal Ed. 2002;86:F108-14.

15. Baschat AA, Harman CR. Antenatal assessment of the growth restricted fetus. Curr Opin Obstet Gynecol. 2001;13:161-8.

16. Bernstein IM, Horbar JD, Badger GJ, et al. Morbidity and mortality among very-low-birth-weight neonates with intrauterine growth restriction. The Vermont Oxford Network. Am J Obstet Gynecol. 2000;182(1 Pt 1):198-206.

17. Zeitlin J, Ancel PY, Saurel-Cubizolles MJ, et al. The relationship between intrauterine growth restriction and preterm delivery: an empirical approach using data from a European case-control study. BJOG. 2000;107:750-8.

18. Yamamoto RM, Francisco RPV, Miyadahira S, et al. Fatores prognósticos para o óbito perinatal em gestações com diástole zero ou reversa na dopplervelocimetria das artérias umbilicais. Rev Bras Ginecol Obstet. 2000;22:353-63.

19. GRIT Study Group. A randomised trial of timed delivery for the compromised preterm fetus: short term outcomes and Bayesian interpretation. BJOG. 2003;110:27-32

20. Francisco RPV, Nomura RMY, Miyadahira S, et al. Diástole zero ou reversa na dopplervelocimetria das artérias umbilicais. Rev Assoc Med Bras. 2001;47:30-6.

21. Andrade JQ, Miyadahira S, Nomura RMY, et al. Dopplervelocimetria dos compartimentos arterial e venoso da circulação fetal e umbilical em gestação de alto-risco: análise dos resultados perinatais. Rev Bras Ginecol Obstet. 2002;24:153-60.
22. Romero R, Kalache KD, Kadar N. Timing the delivery of the preterm severely growth- restricted fetus: venous Doppler, cardiotocography or the biophysical profile? Ultrasound Obstet Ginecol. 2002;19:118-21.

23. Pires P. Aplicações da dopplervelocimetria em gestação de alto risco. In: Pires P. Doppler no $2^{\circ}$ e $3^{\circ}$ trimestres da gestação \& hemodinâmica fetoplacentária. $1^{\mathrm{a}}$ ed. Recife: Edupe; 2006. p. 67-82.

24. Pires P, Faundes A. Dopplervelocimetria na avaliação hemodinâmica materno-fetal. Femina. 2007;35:383-90.

25. Arduini D, Rizzo G. Normal values of pulsatility index from fetal vessels: a cross-sectional study on 1556 healthy fetuses. J Perinat Med. 1990;18 $165-72$.

26. Schwarze A, Gembruch U, Krapp M, et al. Qualitative venous Doppler flow waveform analysis in preterm intrauterine growth-restricted fetuses with ARED flow in the umbilical artery - correlation with short-term outcome. Ultrasound Obstet Gynecol. 2005;25:573-9.

27. Baschat AA, Gembruch U, Reiss I, et al. Relationship between arterial and venous Doppler and perinatal outcome in fetal growth restriction. Ultrasound Obstet Gynecol. 2000;16:407-13.

28. Müller T, Nanan R, Rehn M, et al. Arterial and ductus venosus Doppler in fetuses with absent or reverse end-diastolic flow in the umbilical artery: correlation with short-term perinatal outcome. Acta Obstet Gynecol Scand. 2002;22:786-91.

29. Meyberg GC, Solomayer EF, Grischke EM, et al Does the measurement of four fetal arteries provide more information than the measurement of just two arteries in prenatal Doppler sonography? Ultrasound Obstet Gynecol. 1999;13:407-14.

30. Zelop CM, Richardson DK, Heffner LJ. Outcomes of severely abnormal umbilical artery doppler velocimetry in structurally normal singleton fetuses. Obstet Gynecol. 1996;87:434-8. 